\title{
Research on Resistor-Loaded Half-Ellipse Antenna System for GPR Application
}

\author{
Xueping Li, ${ }^{1}$ Jinjin Shao, ${ }^{2}$ and Yu Zhang ${ }^{1}$ \\ ${ }^{1}$ College of Physics and Electronic Engineering, Henan Normal University, Xinxiang 453007, China \\ ${ }^{2}$ South University of Science and Technology of China, Shenzhen 518055, China \\ Correspondence should be addressed to Xueping Li; lxpslxhhw@126.com
}

Received 3 December 2015; Revised 20 January 2016; Accepted 28 January 2016

Academic Editor: Ikmo Park

Copyright (c) 2016 Xueping Li et al. This is an open access article distributed under the Creative Commons Attribution License, which permits unrestricted use, distribution, and reproduction in any medium, provided the original work is properly cited.

\begin{abstract}
A resistor-loaded half-ellipse antenna system mounted on a vehicle as a candidate for the exploration of the lunar subsurface is investigated. The antenna system includes two identical half-ellipse antennas, one is used for transmission, and the other is for reception. A resistive loading technique for broadening the bandwidth and improving impulse radiation is introduced. The performance of the proposed antenna with different height above ground surface is studied, and the influence of the vehicle on the antenna is analyzed. Then the antenna is manufactured and mounted on a vehicle as some tests are done. The simulated and measured antenna VSWR and radiation patterns are compared together, and good agreements between them are achieved.
\end{abstract}

\section{Introduction}

Ground penetrating radar (GPR) has been widely used for a large number applications, including archaeology, geophysical research, mine detection, and civil engineering [1]. In recent years, more and more researchers pay attention to the lunar and planetary exploration [2]. GPR is a preferred candidate for such missions in particular in the case of the rather small landers because of the extremely severe constraints on mass, power, instrument deployment, operation, and nondestructive detecting technique.

The antenna is one of the most critical components in a GPR system. It has to couple the energy from air to ground, which is lossy and dispersive. Two separate antennas are often used for transmission and reception, as it is difficult to isolate the receiver from the transmitter due to the unavailability of fast switches. In order to distinguish the desired signal of the target object from the random signal, it is very essential that the antenna is capable of transmitting the signal with suppressed late-time ringing. In general, antennas suitable for GPR system can be divided into several types, such as resistively loaded dipole $[3,4]$, bow-tie antenna $[5,6]$, spiral antenna [7], and TEM horn $[8,9]$. In the last couple of decades, bow-tie antennas have been commonly used in many applications because their bandwidth was enlarged and late-time ringing could be suppressed by means of various loading schemes. Reactive (capacitive or inductive) loading has been introduced in $[10,11]$; unfortunately such reactive loading usually realized as gaps or slots in the antenna, if not combined with any form of resistive load, could not be suitable for impulse GPR applications since it usually exhibits a significant level of late-time ringing which might seriously degrade the GPR performance. Reference [12] presented a RC-loaded bow-tie antenna and foam-based absorbers for improving pulse radiation. Comparing with the reactive (capacitive or inductive) loading, such method can reduce the late-time ringing, but the ringing still displays a relatively high level. In [13], a cavity-backed resistively loaded bowtie antenna has been investigated by using FDTD, including optimal resistive loading and near-field performance, but there is no attention to the input impedance and radiated waveform. Furthermore, most bow-tie antennas used in GPR systems have triangular shape arms. However, the impedance of this kind of antenna always has a high impedance about $200 \Omega$ that is difficult in matching with the $50 \Omega$ coaxial cable in the absence of an impedance transformer [14]. 
In view of the above-mentioned problem, in this paper a resistor-loaded half-ellipse antenna system mounted on a vehicle as a candidate for the exploration of the lunar subsurface is proposed. The antenna system includes two identical half-ellipse antennas. Each antenna has a pair of half-elliptical-shape arms, which is loaded by continuous resistive elements. A rectangular conducting backed cavity is attached above the antenna system to seek good radiation directivity. In order to improve the matching between the antenna and the feed line, two identical half-ellipse arms are used for the antenna element and fed by a pair of coaxial cables whose outer shields are connected to each other for a reference ground, and the inner conductors are connected to each arm of the antenna, respectively; then one balanced feed network with the input impedance of $100 \mathrm{ohm}$ can be obtained. The antenna VSWR and radiation patterns are studied. Then both the effects of antenna height above ground surface and the vehicle to the VSWR and radiated pulse of antenna are all studied. Finally some tests are done in the desert. The tests have well illustrated the performance of the antenna system.

\section{Antenna Design and Structure}

In order to broaden the bandwidth and improve impulse radiation, the antenna is loaded by resistive elements. The $\mathrm{Wu}$-King profile is the most widely used resistive loading technique [3]. Though it has been shown that in this case antenna radiation efficiency may go down to as low as $30 \%$ [15], the efficiency is not the most important issue in applications such as vehicle-mounted GPR for shallow target under enough high power. In such an application, to obtain good fidelity of radiation waveform, low radiation efficiency is also acceptable by the resistive loadings. The loading profile here adopted for half-ellipse antenna is derived from the work of $\mathrm{Wu}$-King. The $\mathrm{Wu}$-King profile can be represented as the resistance per unit length:

$$
R^{i}\left(\frac{z}{L}\right)=\frac{\zeta_{0} \psi_{0}}{2 \pi L(1-z / L)}(\Omega / \mathrm{m}) .
$$

$z$ is the distance along the arms from the drive point, $L$ is the length of the arm, $\zeta_{0}=\sqrt{\mu_{0} / \varepsilon_{0}}=120 \pi \Omega$ is the free space impedance, and $\psi_{0}$ is the complex expansion parameter discussed in [3]. In order to use the Wu-King profile to calculate our planar half-ellipse structure, two equivalent processes are used based on the equal surface area. The planar half-ellipse is equalized to a rectangle and then the rectangle is equalized to a slender rod. After this the $\mathrm{Wu}-$ King profile can be easily implemented in our design. In this paper, $\psi_{0}=1.1$, the input impedance of the antenna is about $100 \Omega$. The antenna resistive loading is technically performed by realizing multiple equally spaced concentric slots having fixed width $2 \mathrm{~mm}$. Referring to the Wu-King profile and the actual resistance distribution table, these slots are bridged with chip resistors $R^{i}$ under some adjustment and optimization of processing, whose resistance value is, respectively, $10 \Omega, 12 \Omega, 15 \Omega, 20 \Omega, 26 \Omega$, and $44 \Omega$ from the drive point. Each chip resistor $R^{i}$ along the slot is formed by five parallel resistors.

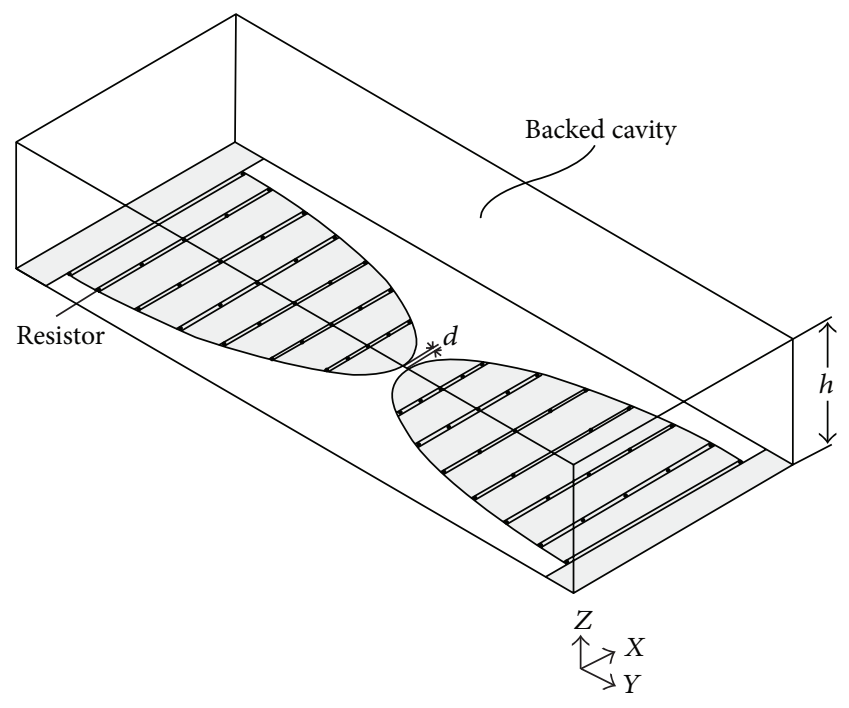

FIGURE 1: Geometry of the resistor-loaded HEA.

The geometry of the proposed antenna can be seen in Figure 1. The dimensions are as follows: $L=166 \mathrm{~mm}, W=$ $120 \mathrm{~mm}, h=22 \mathrm{~mm}$, and $d=4 \mathrm{~mm}$, where $L$ is the length of the half-ellipse arm, $W$ is the half-ellipse arm width, $h$ is the height of the backed cavity, and $d$ is the space between two half-ellipse arms. Due to mass, volume, and sensing requirements, a backed cavity with height of $22 \mathrm{~mm}$ which could not handicap the moving ability of the vehicle is adopted. The two identical half-ellipse antennas are aligned horizontally next to each other for transmission and reception. The antenna elements inside the rectangular backed cavity are separated by a piece of metal plate to minimize the coupling between them. The antenna system which can operate from $250 \mathrm{MHz}$ to $750 \mathrm{MHz}$ with the central frequency $500 \mathrm{MHz}$ is constructed on a $360 \mathrm{~mm} \times 300 \mathrm{~mm}$ FR4 substrate with thickness of $1 \mathrm{~mm}$ and relative permittivity of 4.4 . The input impedance of the antenna is shown in Figure 2, while one conventional half-ellipse antenna (HEA) and bow-tie antenna having the same size are also given for comparison. From Figure 2, it is indicated that the real part of our antenna is around $100 \Omega$ and varies smoothly compared with HEA and bow-tie antenna. By introducing the resistive loading in the antenna, the antenna exhibits much more broadband characteristics.

The structure of the vehicle whose dimensions are $1.2 \mathrm{~m} \times$ $0.8 \mathrm{~m} \times 0.87 \mathrm{~m}$ is presented in Figure 3. The GPR antenna system is mounted on the bottom of the vehicle, and there is a distance of about $30 \mathrm{~cm}$ from the ground surface.

\section{Simulations and Measurements}

The antenna model has been performed by the commercial electromagnetic simulator CST; then one prototype of the proposed antenna is fabricated as shown in Figure 4. The VSWR and radiation patterns are analyzed, and both the effects of antenna height above ground surface and the vehicle on the VSWR and radiated pulse of antenna are all studied. Finally some tests are done in the desert. 


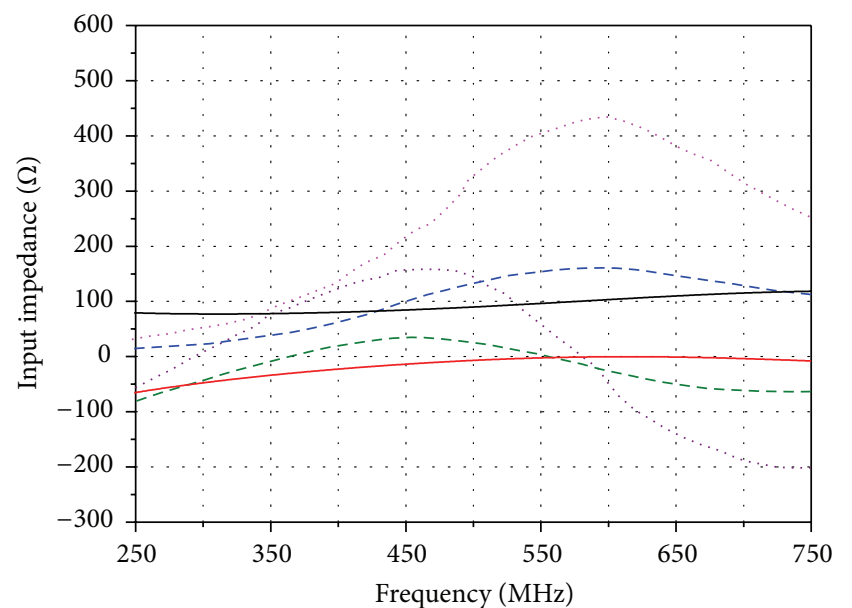

Bow-tie antenna_real

..... Bow-tie antenna_imag

- - - HEA_real

- - - HEA_imag

_ Resistor-loaded HEA_real

— Resistor-loaded HEA_imag

FIGURE 2: Comparison of the input impedance.

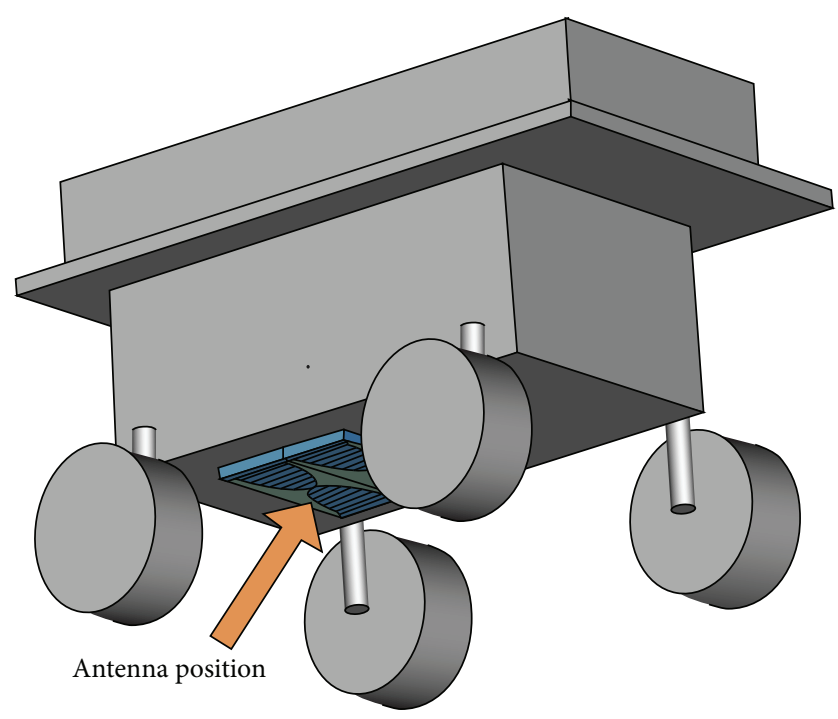

Figure 3: The structure of the vehicle.

The simulated and measured VSWR of the vehicular antenna system is shown in Figure 5. It can be seen that the VSWR is less than 2 over the whole frequency range. The simulated result is well consistent with the measured one. The simulated antenna efficiency is about $13 \%$ to $22 \%$ among the whole frequency range as a great part of the energy provided to the antenna is dissipated by the resistive loadings. In our vehicle-mounted GPR for shallow subsurface target, to obtain good fidelity of radiation waveform, low radiation efficiency is also acceptable by the resistive loadings.

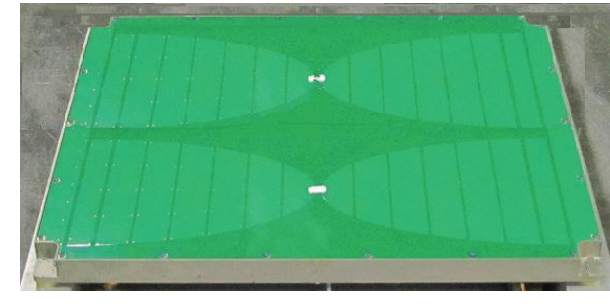

FIgURE 4: Fabricated antenna model.

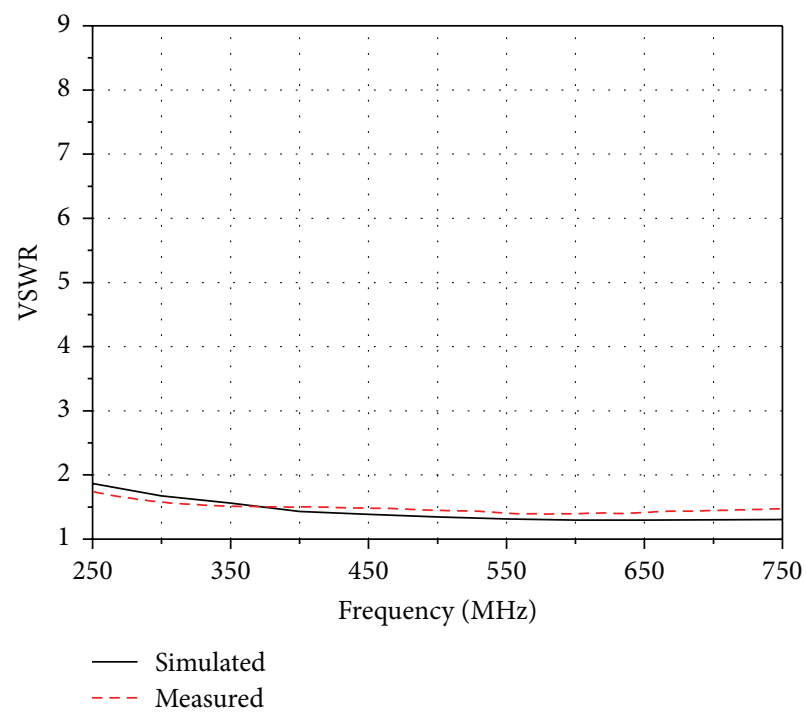

FIGURE 5: Simulated and measured VSWR of the antenna system.

The simulated and measured E-plane radiation patterns at the frequencies of 250,500 , and $750 \mathrm{MHz}$ are shown in Figure 6. It is observed that the antenna has smooth main lobe and good directivity, and the main radiation direction points to the ground. The simulated radiation pattern of the antenna is in good accord with the measured results. The simulated and measured gain of proposed antenna is, respectively, about $-7 \mathrm{dBi}$ and $-7.5 \mathrm{~dB}$ at $500 \mathrm{MHz}$.

The simulated and measured isolation between two antennas are plotted in Figure 7. It is indicated that the isolation is more than $35 \mathrm{~dB}$ over the $250-750 \mathrm{MHz}$ frequency band.

Figure 8 shows the characteristics of VSWR and radiated pulse waveform of the antenna with different heights above ground surface. It can be seen that the VSWR of the antenna attached to the ground surface is much better than that of the antenna elevated above ground $30 \mathrm{~cm}$. The amplitude of the antenna with elevation above the ground surface $30 \mathrm{~cm}$ is about a third of antenna attached to the ground, but both the radiated waveforms can be satisfactory for the impulse GPR application because of little distortion.

To investigate the characteristics of the antenna mounted on the bottom of the vehicle and evaluate the impact of the latter, the antenna and vehicle are simulated and manufactured in the same computational volume. As seen in Figure 9, 


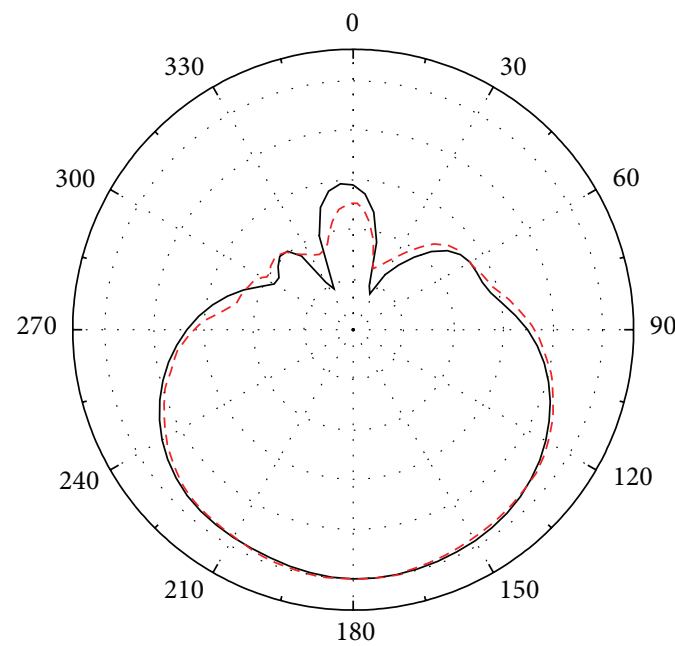

- Simulated

- - - Measured

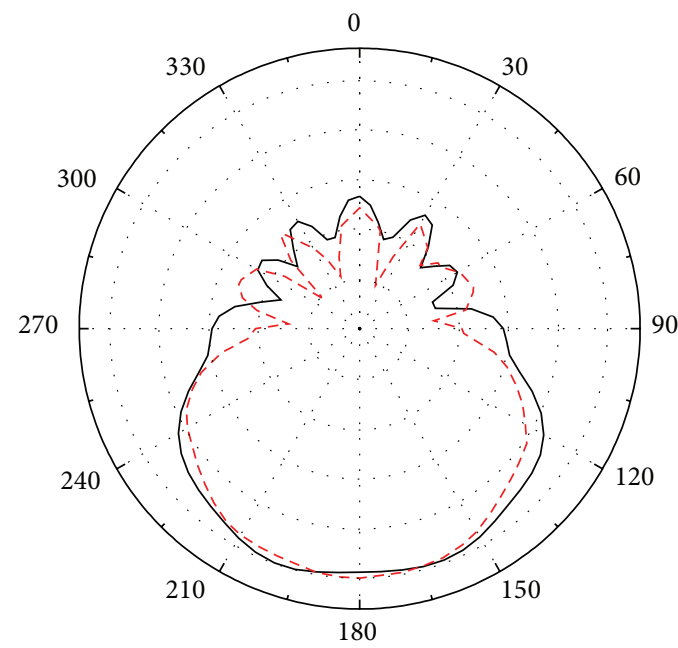

- Simulated

(a)

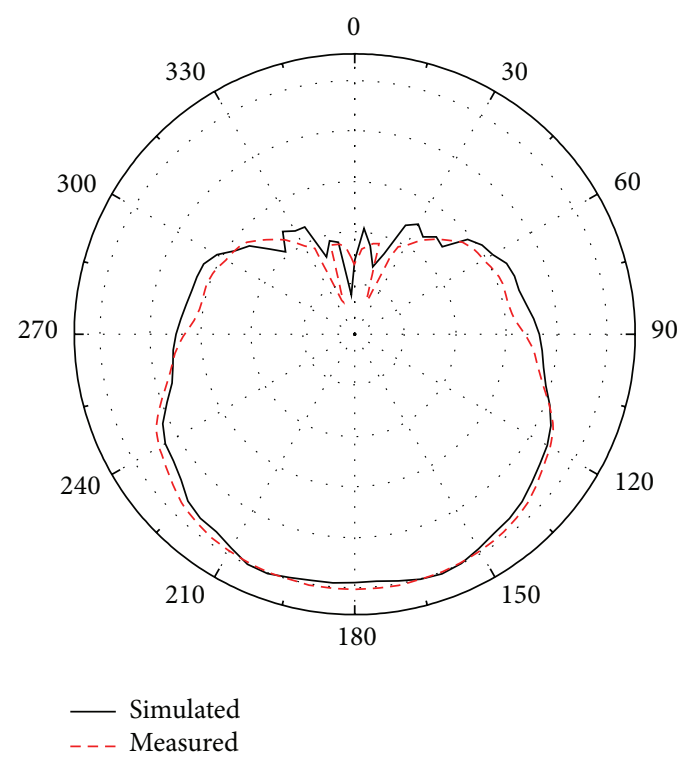

(c)

FIGURE 6: Normalized radiation patterns of E-plane: (a) $250 \mathrm{MHz}$; (b) $500 \mathrm{MHz}$; (c) $750 \mathrm{MHz}$.

the VSWR of the antenna with the vehicle is consistent with that of the antenna without the vehicle, and the influence of the vehicle on the antenna is very little. The existence of the vehicle only makes the late-time ringing of radiated pulse a little larger.

In order to fully validate the performance of the vehiclemounted antenna system, it is applied in our GPR detection. The antenna system is mounted on the bottom of the vehicle. One antenna is directly excited by a balanced pulse generator and radiates electromagnetic wave into the ground, and the other is used for receiving the echoes from the underground targets. As the vehicle moves forward, one continuous set of the backscattered signals including the underground targets will be recorded and the corresponding B-scan can be obtained. Figure 10(a) gives out the experiment scene. Figure $10(\mathrm{~b})$ is the corresponding measured B-scan. A row of uniform reinforced fabric can be found at the arrow and in a relatively good arrangement with the terrain structure.

\section{Conclusion}

In this paper, a resistor-loaded half-ellipse antenna system mounted on a vehicle has been introduced. The antenna prototype is fabricated and installed on the vehicle and then 


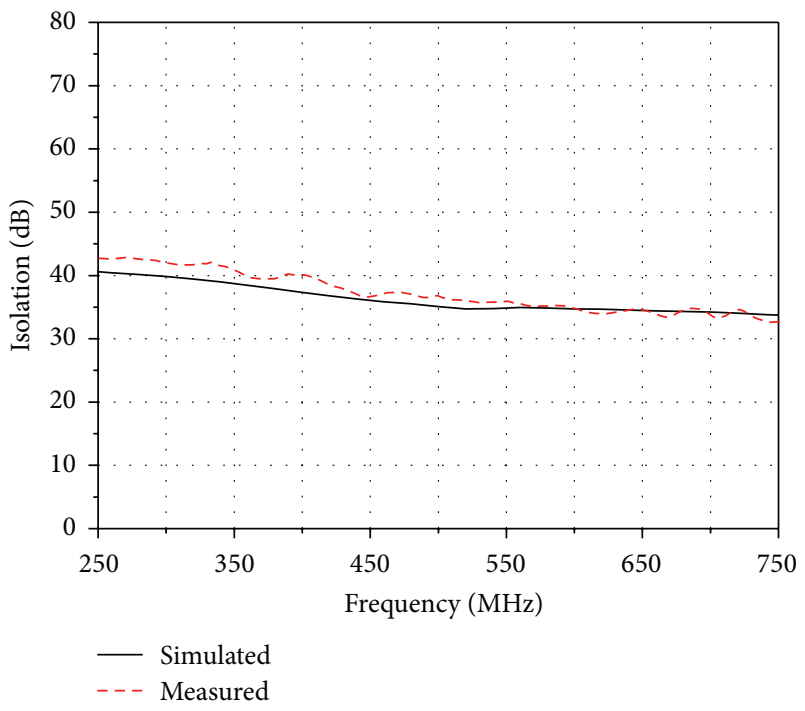

Figure 7: The isolation between the antennas.

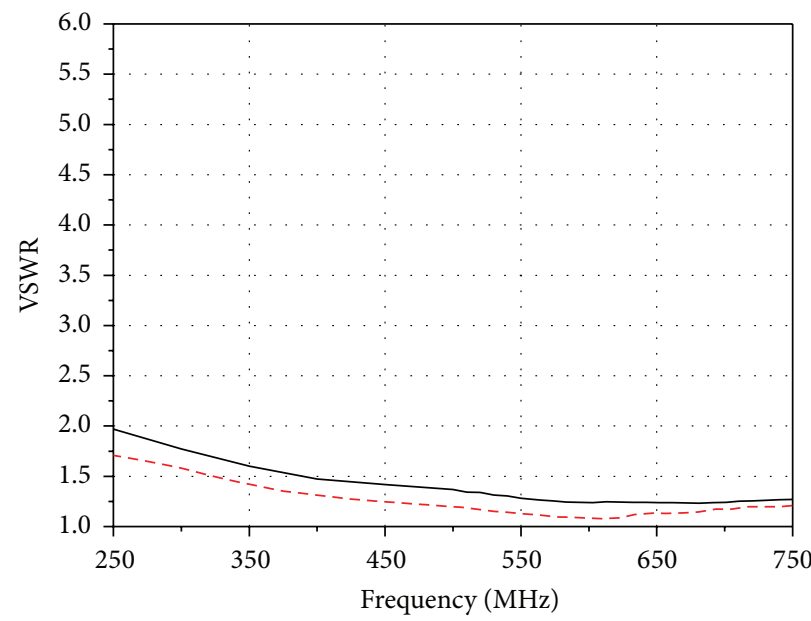

Elevation $=30 \mathrm{~cm}$

- - - Elevation $=0 \mathrm{~cm}$

(a)

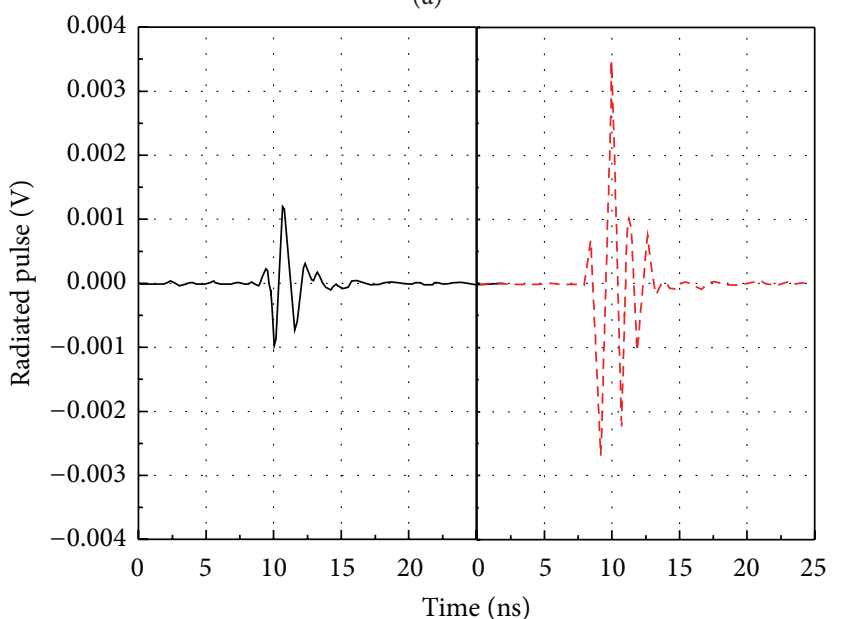

- Elevation $=30 \mathrm{~cm}$

- - Elevation $=0 \mathrm{~cm}$

(b)

FIGURE 8: Comparison of the antenna with different elevation above the ground: (a) VSWR; (b) radiated waveform.

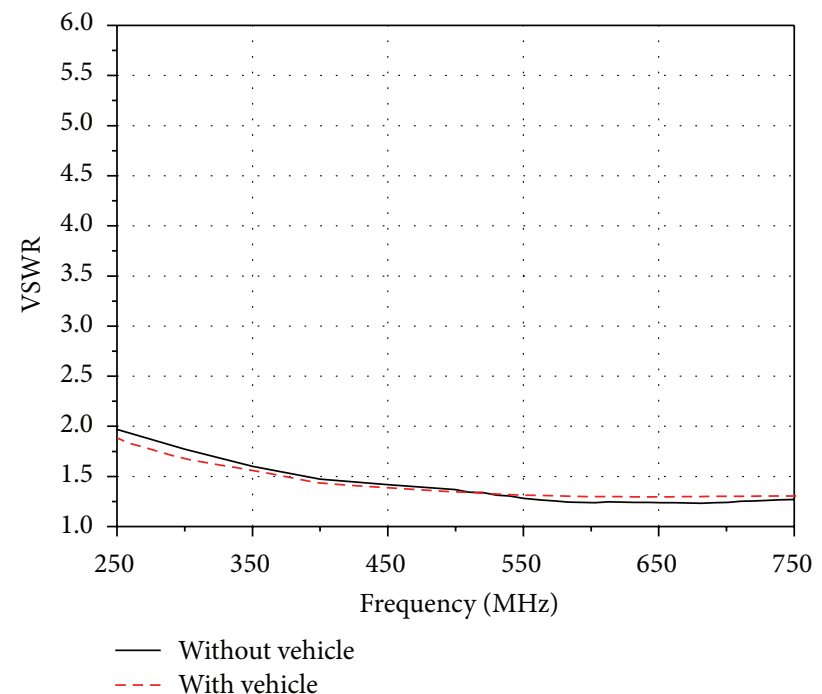

(a)

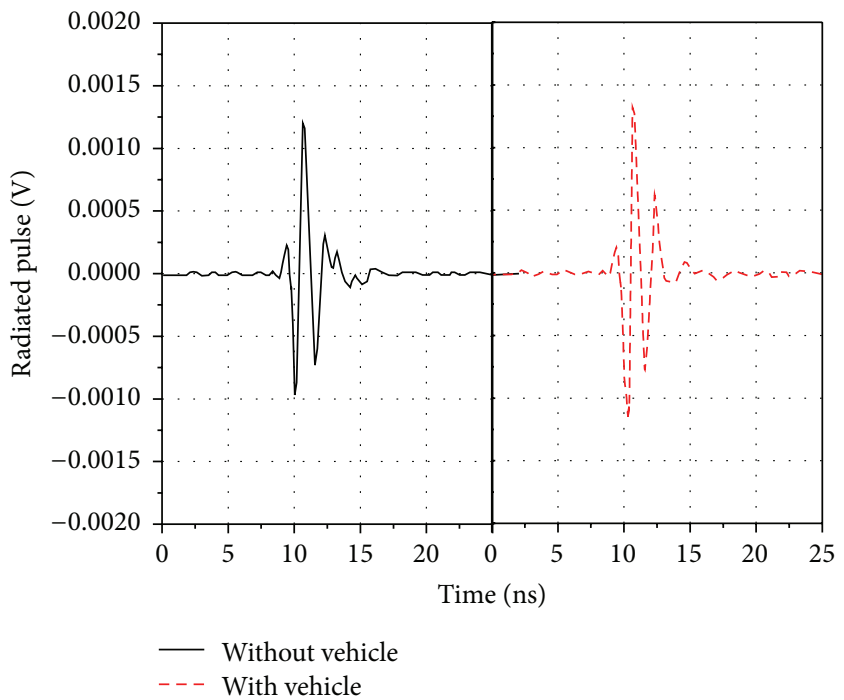

(b)

FIGURE 9: Comparison of resistor-loaded HEA with and without the vehicle: (a) VSWR; (b) radiated waveform.

measured in an anechoic chamber. Both the effects of antenna height above ground surface and the vehicle on the VSWR and radiated pulse of antenna are all studied. Finally some tests are done in the desert showing that the proposed antenna system can be well used in a vehicle-mounted GPR system.

\section{Conflict of Interests}

The authors declare that there is no conflict of interests regarding the publication of this paper.

\section{Acknowledgments}

This work has been supported by the National Basic Research Program of China ("863” Program) of Grant 2012AA061403 


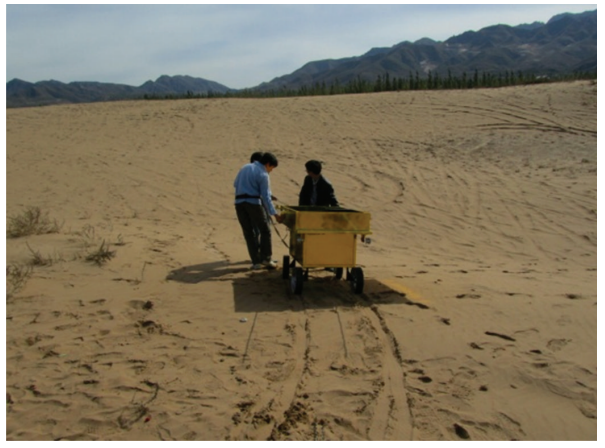

(a)

Trace

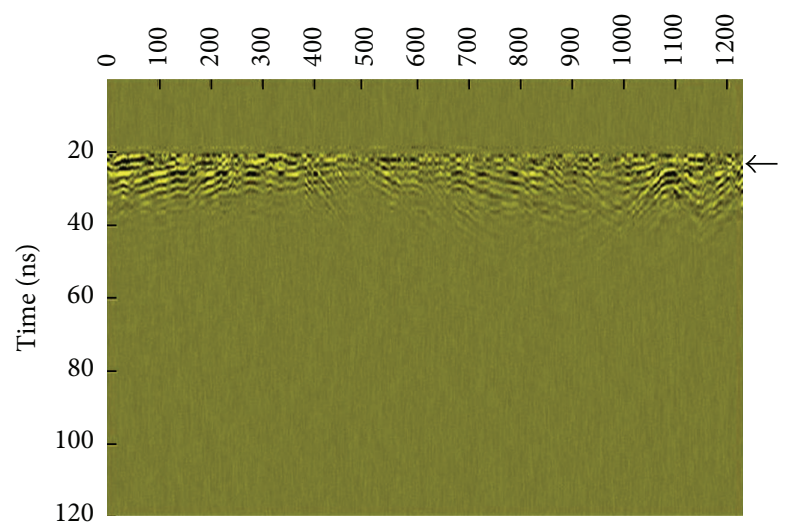

(b)

FIGURE 10: Experiment: (a) experiment scene; (b) radar profiles of desert.

and Doctoral Scientific Research Start-Up Foundation of Henan Normal University of Grant 5101029170276.

\section{References}

[1] D. J. Daniels, Ground Penetrating Radar, IEE, London, UK, 2nd edition, 2004.

[2] T. Kobayashi, S. R. Lee, and J. S. Ping, "Kaguya lunar radar sounder observation of sinus iridum," in Proceedings of the 14th International Conference on Ground Penetrating Radar (GPR '12), pp. 913-916, Shanghai, China, June 2012.

[3] T. T. Wu and R. W. King, "The cylindrical antenna with nonreflecting resistive loading," IEEE Transactions on Antennas and Propagation, vol. 13, no. 3, pp. 369-373, 1965.

[4] A. S. Turk and B. Sen, "Ultra wide band antenna designs for ground penetrating impulse radar systems," in Proceedings of the IEEE International Symposium on Electromagnetic Compatibility (EMC '03), vol. 2, pp. 888-891, Istanbul, Turkey, May 2003.

[5] A. V. Vorobyov, A. G. Yarovoy, P. Aubry, and L. P. Ligthart, "Cavity-backed UWB antenna for impulse radio applications," in Proceedings of the 2nd European Conference on Antennas and Propagation (EuCAP '07), pp. 1-6, Edinburgh, UK, November 2007.

[6] R. C. Hadarig, M. E. de Cos, Y. Alvarez, and F. Las-Heras, "Novel bow-tie antenna on artificial magnetic conductor for $5.8 \mathrm{GHz}$ radio frequency identification tags usable with metallic objects,"
IET Microwaves, Antennas and Propagation, vol. 5, no. 9, pp. 1097-1102, 2011.

[7] H. Nakano, T. Igarashi, H. Oyanagi, Y. Iitsuka, and J. Yamauchi, "Unbalanced-mode spiral antenna backed by an extremely shallow cavity," IEEE Transactions on Antennas and Propagation, vol. 57, no. 6, pp. 1625-1633, 2009.

[8] A. S. Turk and A. K. Keskin, "Ultra wide band TEM horn antenna designs for ground penetrating impulse radar," in 2012 IEEE International Conference on Ultra-Wideband (ICUWB '12), pp. 87-91, Syracuse, NY, USA, September 2012.

[9] A. S. Turk and H. Nazli, "Hyper-wide band TEM horn array design for multi band ground-penetrating impulse radar," Microwave and Optical Technology Letters, vol. 50, no. 1, pp. 7681, 2008.

[10] B. L. J. Rao, J. E. Ferris, and W. E. Zimmerian, "Broadband characteristics of cylindrical antennas with exponentially tapered capacitive loading," IEEE Transactions on Antennas and Propagation, vol. 17, no. 2, pp. 145-151, 1969.

[11] L. L. Liu, Y. Su, C. L. Huang, and J. J. Mao, "Study about the radiation characteristics of bow-tie antennas with discrete resistorloaded," in Proceedings of the Asia-Pacific Microwave Conference (APMC '05), pp. 4-7, IEEE, Suzhou, China, December 2005.

[12] A. A. Lestari, A. G. Yarovoy, and L. P. Lightart, "Capacitivelytapered bowtie antenna," in Proceedings of the Millennium Conference on Antennas and Propagation (AP '00), pp. 1-4, Davos, Switzerland, April 2000.

[13] D. Caratelli, A. G. Yarovoy, and L. P. Ligthart, "Full-wave analysis of cavity-backed resistively loaded bow-tie antennas for GPR applications," in Proceedings of the 5th European Radar Conference (EuRAD '08), pp. 204-207, Amsterdam, The Netherlands, October 2008.

[14] J. J. Shao, C. Chen, J. Chen, Y. C. Ji, G. Y. Fang, and H. J. Yin, "Study of UWB half-ellipse antenna with a shallow backed cavity in vital sign detection," in Proceedings of the 14th International Conference on Ground Penetrating Radar (GPR '12), pp. 89-92, IEEE, Shanghai, China, June 2012.

[15] A. A. Lestari, A. G. Yarovoy, and L. P. Ligthart, "RC-loaded bowtie antenna for improved pulse radiation," IEEE Transactions on Antennas and Propagation, vol. 52, no. 10, pp. 2555-2563, 2004. 


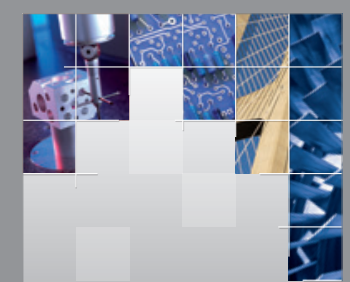

\section{Enfincering}
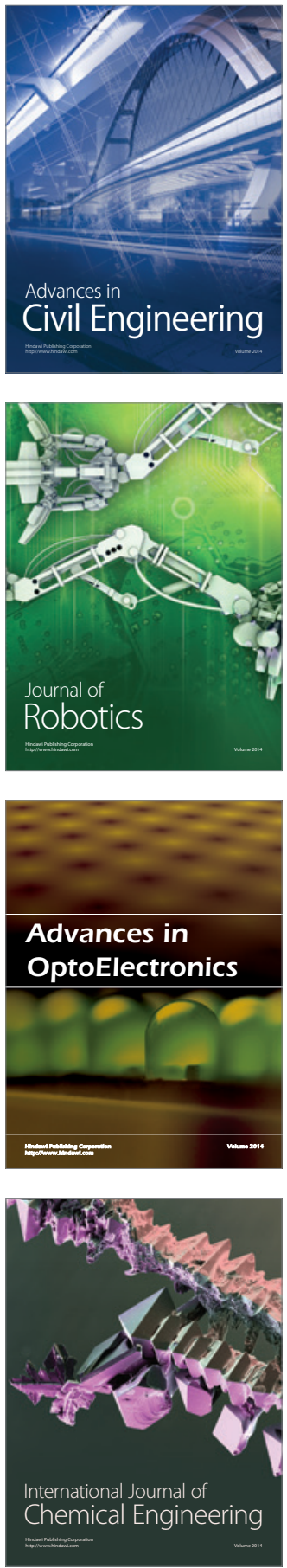

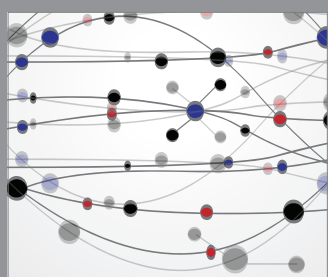

The Scientific World Journal

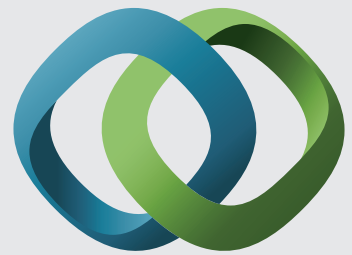

\section{Hindawi}

Submit your manuscripts at

http://www.hindawi.com
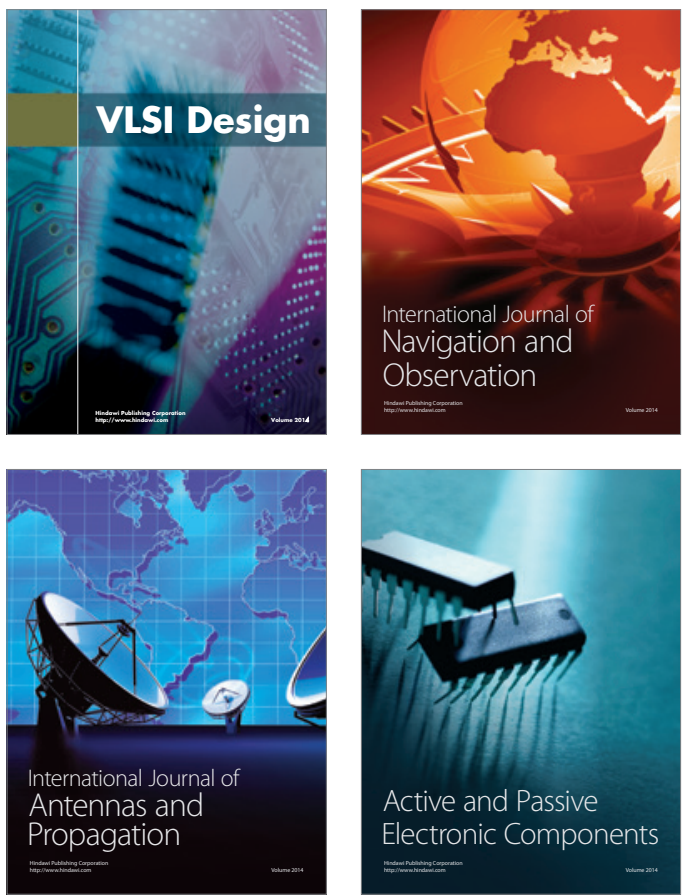
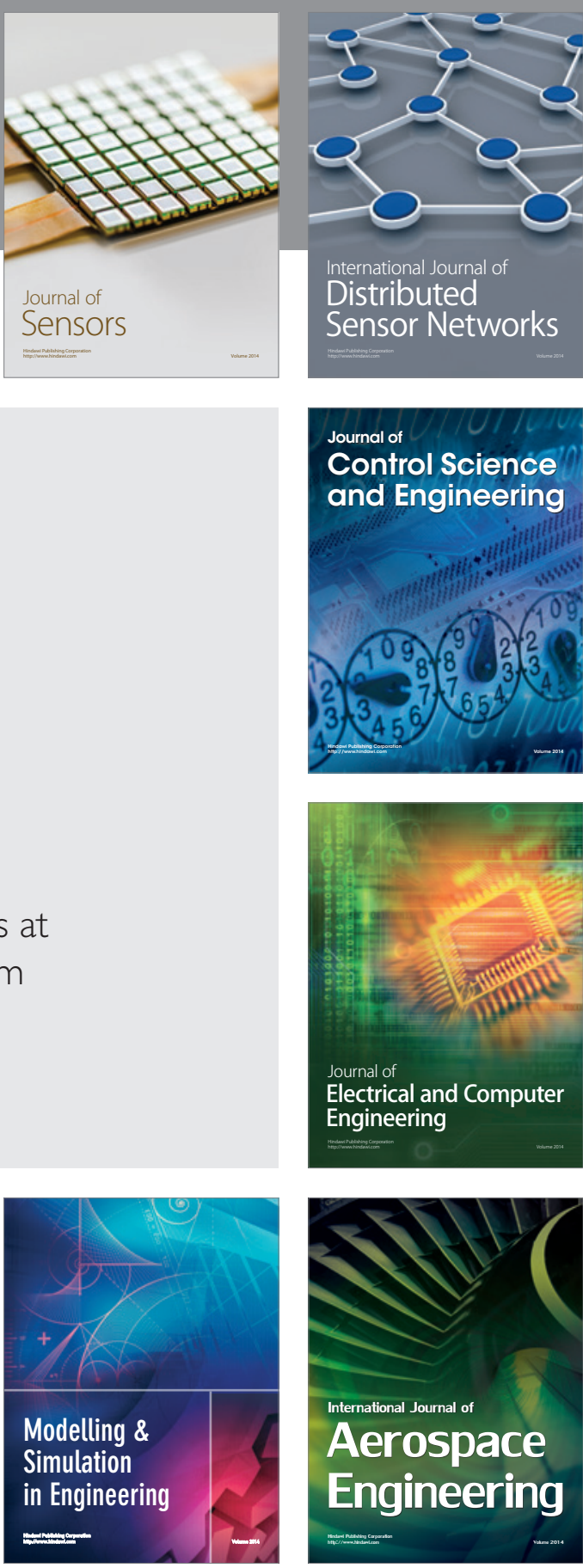

International Journal of

Distributed

Sensor Networks

Journal of

Control Science

and Engineering
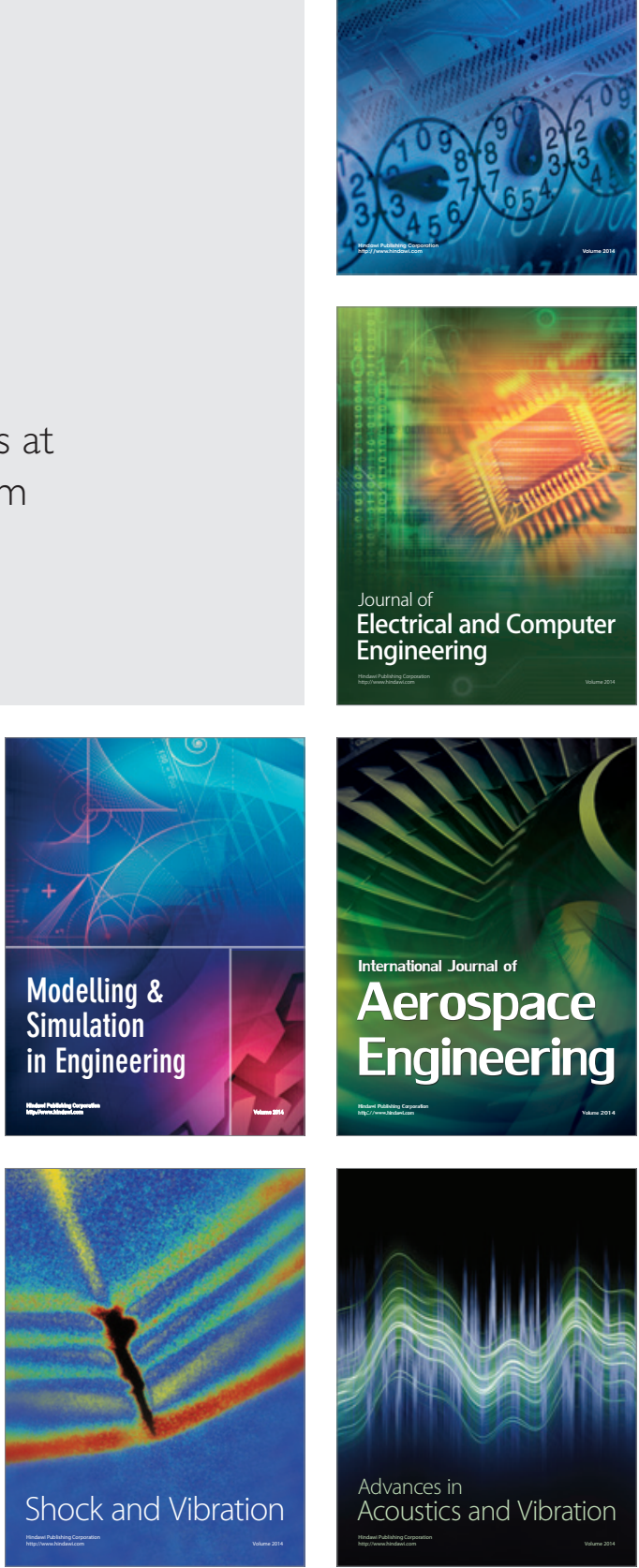